\title{
Avantages et limites d'une technique d'aide à l'explicitation dans la formation des auxiliaires de puériculture : étude exploratoire
}

\author{
Advantages and Limitations of a Technique Using Explicitation Interviews For the \\ Formation of Pediatric Nurse Assistant Students: an Exploratory Study \\ Colette METTÉ', Anne-Marie BESANÇON', Rémi GAGNAYRE', Claire MARCHAND'
}

\begin{abstract}
Résumé Contexte : A partir d'un constat selon lequel certaines élèves auxiliaires de puériculture présentaient des difficultés dans l'acquisition des connaissances déclaratives alors quelles possédaient des connaissances procédurales satisfaisantes, nous nous sommes interrogés sur les possibilités de mobiliser, au cours d'un entretien de tutorat utilisant des techniques d'aide à l'explicitation, les connaissances procédurales de ces élèves dans le but d'améliorer leurs connaissances déclaratives. Buts et méthodes: Ce travail avait pour but d'apprécier les avantages et les limites de la technique d'aide à l'explicitation à partir de l'opinion des élèves et de l'etude de leurs résultats académiques. Résultats : Cette recherche-action à caractère qualitatif a concerné 9 élèves d'une promotion de 42. L'opinion des élèves par rapport à la technique d'aide à l'explicitation est positive. Elle leur a permis de mettre en évidence leurs difficultés d'apprentissage; de mettre en place les actions correctives; de maintenir ou d'améliorer les résultats théoriques. Conclusion : Cette étude semble confirmer des apports constatés par d'autres études : la technique de l'entretien d'explicitation favorise le raisonnement métacognitif, renforce la confiance et facilite la résolution de problèmes en situation professionnelle.
\end{abstract}

Mots clés Formation d'auxiliaires de puériculture ; connaissances déclaratives ; connaissances procédurales ; compétences ; tutorat ; entretien d'explicitation.

Abstract Context: According to a previous report, some pediatric nurse assistant students presented a poor acquisition of declarative knowledge and, in the same time, were strongly equipped in procedural knowledge. We propose that by " mobilizing " procedural knowledge of these students, we can improve their declarative knowledge by using a technique that supports " explicitation " in an interview. Goals and methods: To appreciate advantages and limitations of "explicitation " interview based on students opinion and the examination of their academic performance. Results: This qualitative interactive study required the participation of 9 students out of 42 in this promotion. The opinion of students based on the technique of " explicitation" interview was positive. It enabled them to highlight their difficulties of learning, to prepare a plan of action with a view to improvement and to maintain or to up grade their theoretical results. Conclusion: This study seemed to confirm observations reported in previous studies. The technique used for "explicitation " interview supports metacognitive reasoning, reinforces confidence and facilitates problems solving in diverse professional situations.

Key words Training of pediatric nurse assistant; declarative knowledge; procedural knowledge; competences; tutorial; explicitation interview.

Pédagogie Médicale 2006;7:91-100

1- Laboratoire de Pédagogie de la Santé (UPRES 3412) - U.F.R. Santé Médecine et Biologie Humaine

Léonard de Vinci - 74 rue Marcel Cachin - 93017 Bobigny cedex - France.

Correspondance : Claire MARCHAND - Laboratoire de Pédagogie de la Santé (UPRES 3412) - UFR Santé Médecine et Biologie Humaine Léonard de Vinci - 74 rue Marcel Cachin - 93017 Bobigny cedex - France - Tel : 0148388898 - Télécopieur : 0148387619

mailto:c.marchand@smbh.univ-paris13.fr 


\section{Recherche et Perspectives}

\section{Introduction}

En France, de nombreux élèves quittent le système scolaire secondaire après une classe de troisième ou une orientation en brevet d'études professionnel $(\mathrm{BEP})^{1}$. Après un parcours parfois difficile, caractérisé par l'alternance de périodes de chômage et d'emplois précaires, certains parviennent dans les écoles d'auxiliaires de puériculture.

Les auxiliaires de puériculture collaborent avec les puéricultrices à la surveillance des enfants dans différentes structures telles que les hôpitaux, les crèches ou les haltes garderies. Elles participent aux soins d'hygiène et de confort. Elles aident les enfants ayant perdu leur autonomie ou ne l'ayant pas encore acquise pour l'accomplissement des activités de la vie quotidienne. Elles participent aux activités d'éveil et d'éducation de l'enfant.

La validation de la formation, d'une durée d'environ 1575 heures $^{2}$, comporte une évaluation des connaissances théoriques et pratiques par une épreuve continue et une épreuve finale.

La note d'évaluation continue - confondue de fait avec un score combiné - est constituée par la somme des moyennes obtenues : à quatre devoirs écrits, aux notes de stage et aux notes des mises en situations professionnelles (notes sur 20). Les devoirs écrits évaluent les connaissances théoriques dites " déclaratives ${ }^{3}$ des élèves et leurs capacités d'analyse à partir d'un cas clinique. L'orthographe et l'application des règles de grammaire ne sont pas prises en compte mais les phrases doivent être compréhensibles. Les mises en situations professionnelles évaluent les connaissances d'action dites " procédurales " ${ }^{3}$. Lépreuve finale comprend un devoir anonyme écrit et une mise en situation professionnelle. Les critères d'évaluation du devoir écrit sont identiques à ceux utilisés lors de l'évaluation continue. Pour être admis, les élèves doivent obtenir la note de 60 sur 120 à l'ensemble des épreuves sans note éliminatoire (note inférieure ou égale à 7 sur 30 pour l'épreuve écrite, note inférieure ou égale à 5 sur 20 pour l'épreuve des soins donnés à l'enfant). Depuis plusieurs années, il est constaté que certains élèves auxiliaires de puériculture présentent des difficultés importantes lors des épreuves écrites, alors que parallèlement leurs résultats lors des mises en situations professionnelles sont très satisfaisants. Sur les quatre promotions ayant précédé celle concernée par cette recherche, les résultats du diplôme professionnel montrent que 27 à $46 \%$ de la promotion avaient obtenu une note inférieure à la moyenne du groupe à l'écrit, alors que 53 à $78 \%$ de ces élèves avaient une note supérieure à la moyenne du groupe lors de l'épreuve de mise en situation professionnelle. La majorité de ces élèves réussit correctement les épreuves prati- ques. Les soins sont judicieux, adaptés à la personne et les notes de mises en situation professionnelle sont souvent supérieures à la moyenne du groupe. En revanche, les notes qu'ils obtiennent lors des contrôles théoriques (notes inférieures à la moyenne du groupe) semblent traduire de réelles difficultés à examiner les connaissances déclaratives mises en œuvre dans leurs pratiques professionnelles. D’une manière générale, ces élèves éprouvent beaucoup de difficultés à exprimer leurs idées et à trouver les mots « justes».

Ainsi, ces élèves acquièrent des connaissances procédurales et utilisent des savoirs qu'ils ont du mal à verbaliser et qui sont peut-être parfois insuffisamment conscientisés. Ce manque de conscientisation et de verbalisation des savoirs en action pourrait représenter un frein à la démarche de soins ${ }^{4}$. Cette démarche est une résolution de problèmes qui nécessite de collecter et analyser des données permettant d'identifier un problème de santé, de planifier des soins et de les réaliser et, enfin, de les évaluer pour effectuer un réajustement en cas de nécessité.

Cet article décrit une expérience de tutorat menée auprès d'élèves auxiliaires puéricultrices présentant des difficultés dans leurs apprentissages théoriques au cours d'une année scolaire. Il s'agit d'une étude exploratoire qualitative de type recherche-action puisqu'il est proposé de mettre en place une intervention éducative nouvelle dans un contexte spécifique de formation et d'en apprécier certains effets auprès d'un public cibles. Notre préoccupation principale concerne l'optimisation des pratiques éducatives, en cherchant à mieux comprendre et contribuer à résoudre des problèmes éducatifs délicats ${ }^{6}$ dans un contexte de formation, sans prétention de généralisation ou de transférabilité. Nous émettons l'hypothèse qu'aider ces élèves à verbaliser leurs connaissances procédurales à l'aide d'une technique d'entretien adaptée pourrait leur permettre de prendre conscience des connaissances déclaratives utilisées dans l'action et d'améliorer éventuellement leurs résultats aux épreuves théoriques.

\section{Méthodes}

\section{Objectifs et cadre théorique de l'expérience}

Cette recherche-action à caractère qualitatif vise à apprécier les avantages et les limites d'une technique d'aide à l'explicitation ${ }^{7}$ pour aider les élèves auxiliaires puéricultrices à verbaliser leurs connaissances théoriques et pour leur permettre d'améliorer leurs apprentissages dans ces domaines.

La technique utilisée issue de l'entretien d'explicitation décrit par P. Vermersch ${ }^{7}$ vise la verbalisation d'une action vécue. Le questionnement utilisé par l'interviewer recentre en permanence le discours de l'interviewé sur ce qu'il a fait 
à un moment précis, lui permettant ainsi d'évoquer puis de verbaliser a posteriori des connaissances procédurales, mais aussi les connaissances déclaratives utilisées au cours de l'action décrite. Les buts de l'entretien d'explicitation sont de trois ordres : 1) aider l'interviewer (le tuteur) à s'informer en lui permettant de comprendre, par exemple, les erreurs produites par l'élève ; 2) aider l'interviewé (l'élève) à s'auto-informer, l'explicitation provoquant une prise de conscience de ses propres stratégies et de son fonctionnement intellectuel ; 3) favoriser, par ce moyen, le développement par l'interviewé de ses facultés métacognitives.

Selon Pierre Vermersch ${ }^{8}$, la description d'une action vécue permet d'analyser les éventuelles difficultés d'apprentissage, les causes d'erreurs, mais aussi ce qui constitue la réussite et l'expertise. La mise en œuvre d'un entretien d'explicitation nécessite l'établissement d'un contrat de communication précisant les buts de l'entretien et s'assurant de la volonté de l'interviewé d'y participer, avec la possibilité pour ce dernier d'interrompre l'entretien à tout moment. Ce type de questionnement a montré ses intérêts dans différentes disciplines scolaires (mathématiques, éducation physique et sportive, anglais, etc.). Il est également utilisé pour la formation des enseignants ou encore lors de séances d'analyse de pratique ${ }^{8}$.

\section{Population de l'expérience}

Le recrutement des élèves s'effectue par concours ${ }^{2}$. La promotion concernée par cette recherche a commencé ses études en octobre 2002 et a terminé en novembre 2003. Cette promotion uniquement féminine était constituée de 42 élèves, dont la langue maternelle était le français. Elle était hétérogène par l'âge et le niveau de formation initiale. L'âge moyen était de 24,5 ans. La plus jeune avait 17 ans, la plus âgée 43 ans. La tranche d'âge 20 - 25 ans était la plus représentée (57\% de la promotion). A l'entrée en formation, 9,5\% des étudiants $(\mathrm{n}=4)$ ne possédaient aucun diplôme ; $7 \%$ étaient titulaires d'un Certificat d'aptitude professionnelle (CAP) $(n=3) ; 45 \%$ étaient titulaires d'un Brevet d'études professionnelles (BEP) $(\mathrm{n}=19) ; 38 \%$ étaient titulaires d'un baccalauréat $(\mathrm{n}=16)$ (nota bene: en France, le baccalauréat est le diplôme qui sanctionne la fin des études secondaires).

Les critères d'inclusion dans cette recherche ont été les suivants : l'élève devait avoir obtenu une note inférieure à la moyenne du groupe au premier devoir écrit et une note supérieure à la moyenne du groupe à la première évaluation pratique.

Onze élèves avaient obtenu une note inférieure à la moyenne du groupe au premier devoir écrit. Dix d'entre eux avaient des notes supérieures à la moyenne du groupe en pratique. Parmi ces derniers, neuf ont été volontaires pour participer à cette recherche. Ces élèves ont constitué le groupe cible.

\section{Outils et déroulement de l'étude}

Cette recherche a comporté deux phases : un entretien individuel de type " explicitation " ${ }^{7}$ avec chaque élève et un entretien individuel à distance du précédent pour permettre à l'élève d'exprimer son opinion sur le premier entretien. Ces deux entretiens et leurs analyses ont été réalisés par la même personne (tuteur-chercheur) qui, en revanche, n'a pas participé aux corrections des devoirs écrits.

\section{Premier temps : entretien d'aide à l'explicitation}

Lors de l'entretien, il a été fait référence à une pratique réussie par l'élève. Le tuteur (chercheur) devait amener l'élève à prendre conscience des connaissances tant procédurales que déclaratives utilisées à l'occasion de ce soin ou de cette pratique.

L'entretien durait environ 30 minutes. Il a été effectué en janvier et février 2003, soit 3 à 4 mois après le début de la formation et à la suite du premier devoir (tableau 3). Il se déroulait de la façon suivante :

dans un premier temps, le tuteur demandait à l'élève de laisser revenir, d'évoquer une pratique qu'il avait réussie et de décrire la façon dont il avait procédé au cours de cette pratique. Le questionnement produit par l'interviewer devait aider l'élève à expliciter le plus finement possible les différentes étapes de son action ; dans un deuxième temps, le tuteur demandait à l'élève de réfléchir sur les connaissances mobilisées au cours de cette pratique et il l'aidait en permanence à repérer son raisonnement cognitif par un questionnement adapté (tableau 1).

\section{Deuxième temps : entretien semi-directif}

Les entretiens de type "explicitation » ont été évalués lors d'un entretien semi-directif auprès des élèves concernés. Pendant cet entretien d'une vingtaine de minutes, il s'agissait de recueillir l'opinion des élèves sur l'entretien précédant pour en dégager les effets sur les élèves. Les questions posées étaient les suivantes : "Selon vous, l'explicitation de la pratique vous a-t-elle permis d'améliorer vos résultats théoriques? Si oui, en quoi les entretiens vous ont-ils permis de progresser? Si non, avez-vous une idée des difficultés qui persistent? Que pourriez-vous mettre en place pour améliorer vos résultats?".

L’entretien s'est situé après le troisième devoir écrit permettant ainsi à l'élève d'évaluer la progression de ses performances. 


\section{Recherche et Perspectives}

\begin{tabular}{|l|l|}
\hline \multicolumn{1}{|c|}{$\begin{array}{c}\text { Exemple de questionnement d'explicitation à partir de la description d'une action. } \\
\text { Cette action correspond à la toilette d'un patient alité }\end{array}$} \\
\hline \multicolumn{1}{|c|}{ Interviewer } & \multicolumn{1}{|c|}{ Interviewé } \\
\hline Par quoi avez-vous commencé ? & Je me suis occupé de faire mon chariot. \\
\hline $\begin{array}{l}\text { Comment avez-vous fait ce jour-là pour faire votre } \\
\text { chariot? }\end{array}$ & J'ai réfléchi à ce qu'il fallait mettre dessus. \\
\hline $\begin{array}{l}\text { Quand vous réfléchissiez, qu'est ce que vous faisiez? } \\
\text { Et qu'avez vous fait à ce moment là ? }\end{array}$ & $\begin{array}{l}\text { J'imaginais dans ma tête que j'étais en train de faire } \\
\text { le soin. }\end{array}$ \\
\hline $\begin{array}{l}\text { Comment saviez-vous qu'il fallait faire le chariot de } \\
\text { cette façon ? }\end{array}$ & $\begin{array}{l}\text { J'ai pris les gants, la serviette, la bassine, la couche, } \\
\text { et puis j’ai préparé toutes les affaires personnelles du } \\
\text { patient. }\end{array}$ \\
\hline
\end{tabular}

\section{Modalités d'analyse}

Les deux entretiens ont fait l'objet d'une analyse de contenu ${ }^{7}$.

Cinq catégories, issues des deux entretiens et en lien avec notre recherche, ont été définies :

1) les jugements portés par les élèves sur leurs compétences pratiques par rapport à l'écrit. En comparant leurs compétences dans les domaines pratique et théorique les élèves expriment leurs difficultés dans ce dernier domaine ;

2) la valorisation de la pratique. Les élèves expriment ici en quoi la pratique est satisfaisante, importante, et valorisante ;

3) les difficultés d'ordre affectif exprimées par les élèves. Nous avons repris ici les expressions révélant des émotions liées à l'apprentissage ou à la réalisation d'une tâche ;

4) les difficultés de verbalisation reconnues par les élèves. Nous avons relevé ici les propos témoignant de difficultés à dire, à expliciter son action, son raisonnement, ses connaissances ;

5) les stratégies métacognitives verbalisées par les élèves. Nous avons classé ici les éléments des verbatim des deux entretiens témoignant d'une capacité de l'élève à analyser son propre fonctionnement intellectuel ou la façon dont il s'y prend pour apprendre, se souvenir, résoudre un problème ou conduire une activité . Cette catégorie inclut entre autres la capacité de l'élève à verbaliser son processus d'apprentissage.

Les verbatim des élèves ont été découpés en groupes de phrases significatifs et classés au sein de ces catégories quand ils s'y rapportaient. Par ailleurs, au cours de l'analyse du deuxième entretien, nous nous sommes aussi attachés à apprécier si les élèves étaient capables de repérer les freins à leur apprentissage et quels processus d'amélioration de leur apprentissage ils avaient pu mettre en place. Enfin, sans prétendre identifier ou encore moins démontrer une relation de cause à effet entre l'intervention et les résultats obtenus aux évaluations écrites par cet échantillon, nous avons recueilli ces résultats et présenté les moyennes et médianes obtenues par le groupe cible et l'ensemble de la promotion.

\section{Résultats}

Les résultats sont présentés pour les neuf élèves auxiliaires puéricultrices (AP) ayant participé à la recherche (de AP1 à AP9). Chaque catégorie est illustrée par quelques propos recueillis auprès des élèves (signalée par AP pour 
auxiliaire puéricultrice et numérotée pour identifier l'élève concernée). Les pratiques décrites par les élèves concernées sont présentées dans le tableau 2.

\begin{tabular}{|l|l|}
\hline \multicolumn{2}{|c|}{ Tableau 2 : } \\
\hline \multicolumn{2}{|c|}{ Pratiques décrites par les élèves de l'étude } \\
\hline AP 1 & Activité d'éveil en crèche (pâte à sel) \\
\hline AP 2 & Aide à la toilette \\
\hline AP 3 & Bain d'un nouveau-né \\
\hline AP 4 & Activité d'éveil en crèche (peinture) \\
\hline AP 5 & Toilette complète d'un patient alité \\
\hline AP 6 & Aide à la toilette \\
\hline AP 7 & Aide à la toilette \\
\hline AP 8 & Change d'un lit \\
\hline AP 9 & Toilette complète d'un patient alité \\
\hline Note : AP = auxiliaire puéricultrice \\
\hline
\end{tabular}

Lanalyse des deux entretiens met en évidence les points suivants :

- jugement des compétences par rapport à l'écrit : trois élèves ont souligné leurs difficultés à s'exprimer par écrit, qu'il s'agisse d'un problème de mémorisation (" A l'écrit joublie les choses ...ça sort de mon esprit. (AP2)", "J'ai du mal à utiliser la théorie. (AP7) "), ou d'un blocage à l'écriture ("Je n'arrive pas à écrire. Je vois des mots de la phrase,... des mots qui manquent,... je préfêre ne plus écrire. (AP2)", " J'ai du mal à m'exprimer sur une feuille. (AP3) ";

- valorisation de la pratique : sept élèves se reconnaissaient des capacités d'apprentissage au niveau pratique et disaient se sentir valorisées dans ce domaine ("Si jai vu le geste en pratique tout de suite cela revient. (AP2)", "Une auxiliaire puéricultrice m'a dit que c'était parfait, quill n'y avait rien à redire. (AP3) ", "J'ai de très bonnes relations avec les enfants. (AP5)", "En pratique je suis bien. (AP8)»);

- difficultés liées à l'affectif : cinq élèves ont remarqué qu'elles manquaient de confiance en elles ou étaient stressées par les situations d'apprentissage ("Cela m'a beaucoup stressé, j'ai eu beaucoup de mal à faire ma démarche de soins. (AP2)", "Je n'ai pas suffisamment confiance en moi pour la théorie, c'est pour cela que je ne suis pas très bonne. (AP7)"). Elles ont fait apparaître les difficultés liées à l'affect ("En classe le petit chinois cétait moi, c'est toujours ce que l'on m'a dit. (AP2)", "J'ai peur d'oublier. (AP5)");

- difficultés de verbalisation : six élèves ont pris conscience de leur gêne dans la verbalisation. Elles ont pointé un manque de vocabulaire et se sont senties dans l'incapacité de donner des explications précises ("Je ne sais pas comment expliquer. J'ai du mal à mexprimer, ou je réponds à côté de la question. (AP3)", "Ça ne vient pas. C'est dur à expliquer. Les mots ne viennent pas. (AP5)");

- les stratégies métacognitives verbalisées par les élèves. Neuf élèves ont été capables de verbaliser leur processus d'apprentissage dès le premier entretien ("J'apprends par cour la phrase jusqu'à ce que je la sache et que je n'ai plus besoin de la lire. (AP2)", "J'ai appris en observant. (AP4)", "J'apprends en écrivant plusieurs fois la technique, sil y a des choses que je ne sais pas bien je me les répète plusieurs fois à haute voix, pour voir si c'est bien et après je vérifie. (AP8)", "J'écris chaque soir une technique avant de me coucher. (APG)»).

Au cours des entretiens semi-directifs, la totalité des élèves a exprimé une opinion positive de l'entretien d'explicitation. Ceux-ci disent :

- avoir modifié leur processus d'apprentissage : ("Cela m’a permis de prendre conscience de certaines choses. C'est vrai, en fait je ne réalisais pas que japprenais, c'était parce que je le voyais. C'est en vous le disant que je me suis rendue compte que c'était comme ça que je fonctionnais. (AP5)", "J'arrive mieux à retenir, cela ma permis de voir comment apprendre, jy arrive mieux et je persiste. (APC) ", "J'ai changé ma façon d'apprendre, je me suis enfermée entre 4 murs et là jai pu apprendre. Je ne me donnais pas le temps... je pensais à autre chose. (AP8) ");

- se sentir plus aptes à repérer leurs difficultés et à corriger leurs erreurs : ("Cela me permet de voir que si joublie des choses, je peux les faire revenir. (AP4)", "Cela me permet de me remémorer, de voir où sont mes erreurs. (AP4)", "Je me base par rapport à la dernière fois, jai oublié donc cette fois il faut que je fasse de cette façon, je me dis tiens il manquait cela. (AP9) ", "Il faut que je réfléchisse plus parce que je ne fais pas assez attention à ce que je fais. (AP7) "); - se sentir plus à l'aise dans l'expression orale ou écrite : 


\section{Recherche et Perspectives}

"Je me suis améliorée par rapport à l'expression. (AP9)", "Je sais mieux expliquer. (AP5)", "J'arrive mieux à formuler. (AP1)", "J'arrive à mieux écrire ce que je pense dans ma tête... l'ecrit n'est plus inaccessible. (AP2)»);

- avoir pris confiance en elles: ("Vous m’avez rassurée. Vous m'avez dit que jétais capable de travailler. On ne me l'avait jamais dit... cela m'a donné confiance. (AP8)", "Je suis plus sûre de moi, jai plus de maturité. (AP9)");

- avoir amélioré leur organisation de travail : "Cela aide à clarifier, car on a tellement de choses à faire que l'on ne sait pas comment sorganiser. (AP9)", "Cela nous aide à réfléchir et nous permet de voir comment on travaille. (AP2)", "Je me suis remise en cause et je me suis dit comment je vais faire pour travailler. (APB) ").

Les résultats des devoirs écrits sont présentés dans le tableau 3.

On constate que l'écart qui existait à l'issue du premier devoir entre la moyenne du groupe cible et celle de la promotion diminue sensiblement jusqu'à l'épreuve écrite du diplôme professionnel (tableau 4). Cet écart est passé de $-3,3$ lors du premier devoir à $-1,3$ lors de l'épreuve du diplôme. Le groupe cible semble avoir, en grande partie, comblé son retard.

L'analyse des médianes confirme la diminution de l'écart entre les deux groupes (tableau 5).

Les élèves du groupe cible ont maintenu leurs résultats ou progressé, alors que certains élèves de la promotion ont présenté d'importantes difficultés.

\section{Discussion}

\section{Les limites de l'étude}

Le groupe cible est constitué d'un nombre limité d'élèves ( $n=9)$ qui venaient d'une seule structure de formation, ce qui ne permet pas de généraliser les résultats obtenus mais nous autorise tout de même à dégager des tendances intéressantes pour les pratiques éducatives au sein de l'école.

Tableau 3 :

\section{Résultats aux devoirs écrits des élèves auxiliaires puéricultrices en fonction des phases de l'étude}

\begin{tabular}{|c|c|c|c|c|c|}
\hline Elève & Devoir 1 & Devoir 2 & Devoir 3 & Devoir 4 & $\begin{array}{l}\text { Epreuve écrite } \\
\text { du diplôme }\end{array}$ \\
\hline AP 1 & 11,9 & 9,4 & 11,1 & 9,6 & 7,9 \\
\hline AP 2 & 10,9 & 14,4 & 12,1 & 12,0 & 9,9 \\
\hline AP 3 & 10,8 & 10,1 & 15,0 & 8,5 & 11,7 \\
\hline $\mathrm{AP} 4$ & 11,6 & 9,2 & 10,8 & 8,5 & 8,3 \\
\hline AP 5 & 11,0 & 10,3 & 13,9 & 9,8 & 10,9 \\
\hline AP 6 & 11,3 & 10,4 & 10,0 & 8,7 & 11,7 \\
\hline AP 7 & 10,3 & 9,4 & 10,6 & 9,2 & 9,9 \\
\hline AP 8 & 10,2 & 10,6 & 12,4 & 10,9 & 10,7 \\
\hline AP 9 & 10,0 & 13,8 & 13,0 & 11,6 & 11,8 \\
\hline & \multicolumn{2}{|c|}{$\begin{array}{l}\text { Entretien d'aide } \\
\text { à l'explicitation }\end{array}$} & \multicolumn{2}{|c|}{ Entretien semi-directif } & \\
\hline
\end{tabular}


Tableau 4 :

Résultats (exprimés sous forme de moyennes arithmétiques des notes/scores sur 20) obtenus respectivement par le groupe cible et l'ensemble de la promotion aux différentes épreuves

\begin{tabular}{|c|c|c|c|c|c|}
\hline & Devoir 1 & Devoir 2 & Devoir 3 & Devoir 4 & $\begin{array}{c}\text { Epreuve écrite } \\
\text { du diplôme }\end{array}$ \\
\hline $\begin{array}{c}\text { Moyenne } \\
\text { de la promotion } \\
(\mathbf{n}=\mathbf{4 2})\end{array}$ & 14,2 & 13,1 & 14,4 & 12,0 & 11,6 \\
\hline $\begin{array}{c}\text { Moyenne } \\
\text { du groupe cible } \\
(\mathbf{n}=\mathbf{9})\end{array}$ & 10,9 & 10,8 & 12,1 & 9,9 & 10,3 \\
\hline Ecarts & $-3,3$ & $-2,3$ & $-2,3$ & $-2,1$ & $-1,3$ \\
\hline
\end{tabular}

Tableau 5 :

Résultats (exprimés sous forme de médianes des notes/scores sur 20) obtenus respectivement par le groupe cible et l'ensemble de la promotion aux différentes épreuves

\begin{tabular}{|c|c|c|c|c|c|}
\hline & Devoir 1 & Devoir 2 & Devoir 3 & Devoir 4 & $\begin{array}{c}\text { Epreuve écrite } \\
\text { du diplôme }\end{array}$ \\
\hline $\begin{array}{c}\text { Médiane } \\
\text { de la promotion } \\
(\mathbf{n}=\mathbf{4 2})\end{array}$ & 14,8 & 13,4 & 14,4 & 11,8 & 11,4 \\
\hline $\begin{array}{c}\text { Médiane } \\
\text { du groupe cible } \\
(\mathbf{n}=\mathbf{9})\end{array}$ & 10,9 & 10,3 & 12,1 & 9,6 & 10,7 \\
\hline Ecarts & $-2,9$ & $-3,1$ & $-2,3$ & $-2,2$ & $-0,7$ \\
\hline
\end{tabular}




\section{Recherche et Perspectives}

Les entretiens se sont déroulés à distance de la pratique. Ils se sont donc appuyés sur les souvenirs des élèves qui ont pu être modifiés dans le temps. La mise en évocation nécessaire à l'explicitation de l'action vécue peut être rendue plus difficile si le délai entre l'évocation de l'action et l'action même est trop important ${ }^{7}$. C'est, dans ce cas, la maîtrise de la technique de l'entretien par l'interviewer qui peut être garante de la mise en évocation de l'interviewé.

Par ailleurs, dans le contexte de la recherche, il n'a été possible de mener qu'un seul entretien d'aide à l'explicitation, alors que, le plus souvent, deux à trois entretiens peuvent être nécessaires pour obtenir une véritable évocation facilitant la prise de conscience d'actions " préréfléchies ", c'est-à-dire non encore conscientisée s. ${ }^{8}$. Cette limite est liée à la difficulté d'introduire une nouvelle activité dans un temps de formation particulièrement chargé. De même, il existe un biais classique en termes d'objectivité dans l'analyse des résultats obtenus, de part la double posture de chercheur et de tuteur.

Enfin, les délais entre la pratique, l'entretien d'explicitation et l'entretien d'évaluation n'ont pas été les mêmes pour chaque élève, car dépendant de la disponibilité de chacun. Ces délais peuvent constituer aussi un biais.

\section{Les avantages de la technique d'aide à l'explicitation}

Les résultats obtenus confirment ceux recueillis auprès de publics différents ${ }^{8}$. Dans cette étude, l'entretien d'explicitation a permis aux élèves : de prendre conscience de leurs connaissances ; de mettre en évidence les difficultés d'apprentissage; de mettre en place les actions correctives ; de maintenir ou d'améliorer les résultats théoriques.

Il donne à l'élève la possibilité de s'observer et de revenir sur une action accomplie lui permettant non seulement de prendre conscience de ses difficultés, mais aussi de ses capacités. Il peut ainsi modifier la perception qu'il a de sa compétence à apprendre : " un feedback sur l'effort que des élèves ont fourni pour accomplir une tâche améliore l'opinion qu'ils ont de leur compétence à accomplir cette tâche $»^{10}$.

De nombreux auteurs s'accordent à considérer que le langage représente une aide précieuse à la conceptualisation $^{11,12,13}$. En permettant à l'élève de verbaliser ses connaissances et ses difficultés, l'entretien d'aide à l'explicitation lui redonne confiance et le repositionne en tant qu'acteur de sa formation. En analysant le soin effectué et en en décrivant la procédure, l'élève retrouve son erreur, en prend conscience, l'analyse et débute une réflexion corrective $^{14}$. Il participe directement à sa formation. Il se sent responsable de son apprentissage et cherche des moyens pour l'améliorer. Il reprend confiance en lui. Sa motiva- tion est renforcée. Après cet entretien, les élèves ont planifié leur apprentissage, ils ont commencé à s'auto-évaluer et à gérer leurs temps ${ }^{15}$.

Les élèves ont été sélectionnés pour leurs grandes difficultés à l'écrit lors du premier devoir. Sans tenir compte dans cette recherche des difficultés de passage à l'écriture que peuvent ressentir les personnes concernées, notre expérience indique que ces élèves auraient probablement présenté des difficultés plus grandes lors des devoirs suivants sans l'apport de l'entretien d'explicitation. En effet, en s'appuyant sur la description d'une action réussie, l'entretien les a mis en confiance et les a incités à persévérer dans la tâche à accomplir et à utiliser l'erreur comme indicateur d'apprentissage. Il semble que la technique d'aide à l'explicitation a été un exercice d'entraînement efficace, qui a appris aux élèves à repérer et à verbaliser les connaissances utilisées. Ces apprentissages pourraient leur être utiles pour passer de la phase d'analyse, où l'élève doit rechercher dans ses connaissances les éléments nécessaires à la compréhension d'une situation, à la démarche de soins ${ }^{4}$. Dans notre étude, la technique d'entretien utilisée semble permettre de maintenir les résultats du groupe cible mais une recherche sur un échantillon plus grand sera nécessaire pour confirmer ce résultat. Cependant, compte tenu de la perception positive émanant de cette expérience sur un petit groupe, et dans un souci d'équité, il nous apparaît nécessaire de rendre accessible cette pratique au plus grand nombre d'élèves de cette école, en attendant la validation d'un lien réel entre l'aide à l'explicitation et les résultats académiques.

\section{Les limites de la technique d'aide à l'explicitation}

Une des limites de ce type d'entretien est d'ordre affectif. Une élève a eu de nombreuses difficultés à verbaliser l'action. La situation d'entretien direct avec le tuteur (et chercheur) paraissait déclencher un sentiment d'angoisse limitant ses capacités d'expression. Cette gêne peut être liée au rapport hiérarchique existant entre l'élève et le tuteur, ce dernier étant à la fois enseignant et évaluateur du même élève, mais aussi à un vécu négatif de l'élève vis-à-vis de la scolarité précédente qui, en provoquant peur, honte, souffrance, sentiments d'échec, rend difficile la conduite vers une conscience réflexive ${ }^{16}$.

Ainsi, il n'est pas toujours possible de mener des entretiens d'explicitation. En fonction des élèves, de leur vécu et de leurs difficultés présentes, mais aussi du degré de maîtrise de la technique par l'interviewer, l'entretien peut s'avérer difficile. L'accord de l'élève et sa participation sont indispensables. Le contrat de communication initial est essentiel et doit être réitéré plusieurs fois au cours de l'entretien ${ }^{8}$. 
L'entretien d'aide à l'explicitation fait appel au langage. Le public étudié possédait au départ une faible maitrise de la langue française lors de l'expression orale mais surtout lors de l'expression écrite. Malgré ce handicap, les élèves ont participé et amélioré leurs performances, ce qui montre que, même en l'absence d'une maîtrise de la langue, l'entretien d'aide à l'explicitation permet d'améliorer l'apprentissage. D'autre part, nous avons constaté que les entretiens avaient permis d'améliorer la maîtrise de la langue en entrânant les élèves à verbaliser leurs actions, à décrire le contexte. La difficulté du passage à l'écriture pouvant être ressentie par les élèves n'a pas été traitée dans cette étude et pourrait faire l'objet d'un entretien d'explicitation spécifique.

La formation des auxiliaires de puériculture est une formation professionnelle nécessitant l'acquisition de connaissances procédurales et déclaratives. Les élèves devront, au cours de leur formation puis durant leur vie professionnelle, passer d'une démarche de novice à un raisonnement d'expert. Ils participeront à leur tour à la formation d'élèves en les encadrant durant leurs stages. Cette démarche s'appuie sur un raisonnement métacognitif, mais aussi sur le passage de la régulation externe, où l'expert régule l'activité du novice de l'extérieur, à la régulation interne, où le novice intériorise peu à peu les compétences pour gérer ses taches de façon autonome ${ }^{17}$. C'est ainsi que l'entretien d'explicitation apparait comme un bon moyen de développer les capacités métacognitives des élèves auxiliaires de puériculture.

\section{Conclusion}

A partir de l'explicitation d'une pratique réussie, les élèves auxiliaires de puériculture ont verbalisé leurs connaissances, identifié leurs difficultés d'apprentissage et mis en place des actions correctives correspondant à leurs besoins propres.

Lexplicitation des pratiques a renforcé le travail des élèves en donnant un sens à leurs apprentissages.
Certains élèves ont souligné que l'explicitation des pratiques avait renforcé leur confiance en pointant des capacités qu'elles ne soupçonnaient pas. Ces élèves se sont réappropriées leur travail et se sont senties responsables de leurs résultats. Le ressenti des élèves confirme la notion énoncée par $\mathrm{P}$. Vermersch ${ }^{18}$, selon laquelle « la mise à jour des démarches a pour but la prise de conscience de ces propres outils intellectuels ».

Cette technique semble donc adaptée à la formation du soignant. Elle devrait pouvoir être utilisée dans les autres formations paramédicales. En effet, un soignant doit non seulement posséder des connaissances, mais aussi prendre des décisions dans des contextes très différents. Il s’agit de passer du raisonnement de novice à la démarche d'expert, qui nécessite un "bagage " de connaissances spécifiques, élaborées, organisées et une capacité de résolution de problèmes. En proposant un retour et une réflexion sur l'action, l'entretien d'explicitation favorise cette démarche.

La technique de l'entretien d'explicitation nécessite d'être bien maîtrisée par les membres de l'équipe pédagogique. Pour cela, les enseignants doivent être formés. Par ailleurs, ils pourront être amenés à utiliser l'explicitation comme support d'analyse de leur propre pratique d'enseignement dans le but d'en mesurer l'efficacité afin de réajuster leurs actions.

\section{Contributions}

Colette Metté a supervisé les aspects méthodologiques du travail et rédigé le manuscrit de l'article. Anne-Marie Besançon a réalisé l'étude dans le cadre d'un mémoire de Maîtrise Universitaire de Pédagogie des Sciences de la Santé, Bobigny, Université Paris 13 (Année 2003-2004). Rémi Gagnayre a révisé le manuscrit de l'article. Claire Marchand a dirigé le travail mémoire dont est issu l'article et a participé à la rédaction de l'article. 


\section{Recherche et Perspectives}

\section{Références}

1. Ministère de l'éducation nationale. Note d'information 02-50. Les diplômes de l'enseignement technologique et professionnel, session 2001.

2. Arrêté du 22 juillet 1994 relatif au certificat d'aptitude aux fonctions d'aide-soignant et au certificat d'aptitude aux fonctions d'auxiliaire de puériculture. Journal Officiel de la République française, 24 juillet 1994.

3. TardifJ. Pour un enseignement stratégique, l'apport de la psychologie cognitive, Montréal : Editions Logiques, 1992.

4. Amar B, Gueguen JP. Soins Infirmiers : Concepts et théories démarche de soins,. Paris : Edition Masson, Collection Nouveaux cahiers de l'infirmière, 1999.

5. Dortier JF (Coord.). Dictionnaire des sciences humaines. Paris : Edition Sciences Humaines, 2004.

6. Landsheere (de) V. L'éducation et la formation, Paris : PUF (coll. Premier cycle), 1992.

7. Vermersch P. L'entretien d'explicitation (3eéd.). Paris : Editions ESF, 2000.

8. Vermersch P, Maurel M. Pratiques de l'entretien d'explicitation, Paris : Edition ESF, collection Pédagogies, 1997.

9. Giordan A, de Vecchi G. Les origines du savoir. Des conceptions des apprenants aux concepts scientifiques (2e ed.). Lausanne : Éditions Delachaux et Niestlé (Collection Actualités pédagogiques et psychologiques), 1994.
10. Schunk DH. Effects of effort attributional feedback on children's perceived self-efficacy achievement. J Educ Psychol 1982;74:548-56.

11. Vygotsky L. Pensée et langage, traduction de Françoise Sève. Paris : Messidor, Édition Sociale, 1985.

12. Gardner H. Histoire de la révolution cognitive, la nouvelle science de l'esprit. Paris : Payot, 1993.

13. Vergnaud G. Au fond de l'action, la conceptualisation. In : Savoirs théoriques et savoirs d'action, Barbier JM (Dir.). Paris : PUF (Pédagogies d'aujourd'hui) 1996:275-92.

14. Novak JD, Gowin DB. Learning how to learn (4th ed.). New York: Cambridge University Press, 1989.

15. Viau R. La motivation en contexte scolaire; Bruxelles : De Boeck-Wesmael, 2003.

16. Faingold N. Les formateurs-tuteurs : Quelles pratiques? Quelle identité professionnelle? In :Altet $M$, Paquay L, Perrenoud P. Formateurs d'enseignants: quelle professionnalisation? Bruxelles : De BoeckWesmael (Collection perspective en éducation). 2002:193-218.

17. Meirieu P. Transmettre oui mais comment? Sciences Humaines 2002;36:40-5.

18. Vermersch P. Conscience directe et conscience réfléchie. Expliciter, Journal du groupe de Recherche sur l'explicitation. 2001;39:10-31.

Manuscrit reçu le 7 février 2005 ; commentaires éditoriaux formulés aux auteurs le 27 mai 2005 ; accepté pour publication le $1^{\text {er }}$ mars 2006. 